\title{
La aspirina no fue eficaz en la reducción de eventos cardiovasculares en una población con bajo indice tobillo-brazo
}

\author{
Aspirin was not effective for reducing cardiovascular events in a lo ankle brachial index population
}

Fowkes F. y col. JAMA 2010;303:841-848.

\begin{abstract}
Objetivos
Determinar la efectividad de la aspirina en prevenir eventos cardiovasculares en personas de la población general con un bajo índice tobillo-brazo (ITB) identificado por rastreo.

\section{Diseño, lugar y pacientes}

Este ensayo clínico controlado, aleatorizado y doble ciego* fue efectuado entre Abril de 1998 y Octubre del 2008 en Lanarkshire, Glasgow y en Edinburgo, Escocia central. Incluyó hombres y mujeres entre 50 y 75 años de edad, sin historia de enfermedad vascular. Se invitó a participar a 165.795 voluntarios, de los cuales se rastreó la presencia de enfermedad vascular periférica $($ ITB $<0,95)$ en 28.980 . Finalmente, se aleatorizó en forma computada, consecutivamente y en bloques* de ocho, a 3.330 participantes.
\end{abstract}

Intervención y medición de los resultados principales La población fue aleatorizada a recibir $100 \mathrm{mg}$ de aspirina/día $(n=1.675)$ o placebo $(n=1.675)$. El punto final primario combinado fue la primera ocurrencia de muerte, infarto de miocardio, accidente cerebrovascular (ACV) o revascularización. El análisis se realizó según intención de tratar*.

\section{Resultados principales}

Las características basales de ambos grupos fueron similares. El $72 \%$ de los participantes eran mujeres. La media del ITB fue de 0,86 (DS 0,09). La media de seguimiento de los participantes fue de 8,2 años. Se perdieron diez pacientes $(0,3 \%)$ en total.

Al menos 357 participantes sufrieron un evento final primario (13,5 por cada 1.000 personas/año, IC $95 \%$ 12,2 a 15,0). No hubo una diferencia estadísticamente significativa en la tasa de eventos a lo largo del tiempo entre ambos grupos. Los resultados principales pueden verse en la tabla 1.

Tabla 1: Tasa de eventos principales y de mortalidad global en grupo Aspirina y Placebo

\begin{tabular}{l|c|c} 
& Aspirina & Placebo \\
\hline Tasa de eventos principales $^{\mathrm{a}}$ & $13,7(11,8$ a 15,9$)$ & $13,3(11,4$ a 15,4$)$ \\
\hline Tasa de mortalidad global $^{\mathrm{a}}$ & $12,8(11,0$ a 14,8$)$ & $13,5(11,6$ a 15,6$)$ \\
\hline
\end{tabular}

a Tasa cada 1000 personas/año. Entre paréntesis IC95\%.

\section{Conclusiones}

En pacientes con un ITB menor o igual a 0,95 sin antecedentes de enfermedad CV, la aspirina no se diferenció del placebo en la reducción del punto final primario combinado de muerte, infarto de miocardio, ACV o revascularización.

Fuente de financiamiento: Bayer HealthCare, Sanofi-Aventis, Bristol Myers Squibb.

\section{Comentario}

El índice tobillo brazo es una prueba sencilla y relativamente económica para confirmar la sospecha de obstrucción arterial de los miembros inferiores. Se realiza obteniendo el cociente del valor de la presión arterial sistólica (registrado mediante un doppler portátil) entre las arterias pedias o tibial posterior y la arteria humeral. Dado que normalmente la presión sistólica es mayor en los miembros inferiores, se considera normal un ITB $>$ a 0,9 . Un ITB $<0,9$ tiene una sensibilidad $^{*}$ del $95 \%$ y una especificidad ${ }^{*}$ del 100 para detectar una obstrucción arterial $>50 \%$ en pacientes con claudicación intermitente ${ }^{1}$.

Este ensayo clínico presenta ciertos aspectos que son interesantes de remarcar. Primero, estaba compuesto en un $72 \%$ por mujeres, lo cual debe ser tenido en cuenta a la hora de extraer conclusiones acerca del rol de la aspirina en la prevención de eventos en los hombres. En segundo lugar, hubo una sobreestimación inicial del rol de la aspirina en la reducción de los eventos, estimada en un $25 \%$. Esto llevó a suspender el ensayo meses antes de su finalización estimada al no encontrar diferencias entre ambos grupos, y también debido a una tendencia a mayor cantidad de eventos hemorrágicos mayores con aspirina (HR 1,71; IC95\% 0,99 a 2,97). Tercero, los pacientes que no tenían una adecuada adherencia al tratamiento, independientemente de si tomaban aspirina o placebo, presentaron una tasa de eventos superior al $60 \%$ en comparación con los que manifestaron adherencia. Esto podría implicar que otros factores ajenos al estudio aumentaron la tasa de eventos. Finalmente, debido a que el ITB cumple los criterios de Frame y Carlson para rastreo de enfermedad vascular, nuevos ensayos utilizando otras drogas, como por ejemplo estatinas, podrían ser efectuados en el campo de la prevención de eventos cardiovasculares.

\section{Conclusiones del comentador}

Debido a la evidencia obtenida, por el momento no debería utilizarse la aspirina como profilaxis de eventos cardiovasculares en personas sin clínica ni antecedentes de este tipo por el sólo hecho de presentar un ITB bajo.

Francisco Romeo [ Servicio de Cardiología del Hospital Italiano de Buenos Aires. francisco.romeo@ hospitalitaliano.org.ar ]

Romeo F. La aspirina no fue eficaz en la reducción de eventos cardiovasculares en una población con bajo índice tobillo-brazo. Evid Act Práct Ambul. Jul-Set 2012;15(3):92. Comentado de: Fowkes F, Price J, Stewart M y col. Aspirin for prevention of cardiovascular events in a general population screened for a low ankle brachial index: a randomized controlled trial. JAMA 2010;303:841-848. PMID 20197530.

Referencias

1. Mohler ER, Mitchell E. Noninvasive diagnosis of arterial disease. In: UpToDate, Basow, DS (Ed), UpToDate, Waltham, MA, 2012. 